MATHEMATICS OF COMPUTATION

Volume 81, Number 278, April 2012, Pages 839-860

S $0025-5718(2011) 02534-2$

Article electronically published on September 20, 2011

\title{
THE GENERALIZED POLARIZATION TENSORS FOR RESOLVED IMAGING PART II: SHAPE AND ELECTROMAGNETIC PARAMETERS RECONSTRUCTION OF AN ELECTROMAGNETIC INCLUSION FROM MULTISTATIC MEASUREMENTS
}

\author{
HABIB AMMARI, HYEONBAE KANG, EUNJOO KIM, AND JUNE-YUB LEE
}

\begin{abstract}
This paper deals with the problem of reconstructing the electromagnetic parameters and the shape of a target from multi-static response matrix measurements at a single frequency. The target is of characteristic size less than the operating wavelength. Using small-volume asymptotic expansions of the measurements of high-order, we show how the electromagnetic parameters and the equivalent ellipse of the target can be reconstructed. The asymptotic expansions of the measurements are written in terms of the new concept of frequency dependent polarization tensors. Moreover, we extend the optimization approach proposed in Part I to image geometric details of an electromagnetic target that are finer than the equivalent ellipse. The equivalent ellipse still provides a good initial guess for the optimization procedure. However, compared to the conductivity case in Part I, the cost functional measures the discrepancy between the computed and measured high-order frequency dependent polarization tensors rather than between the generalized polarization tensors. The main reason for such a modification of the cost functional is the fact that the (measured) frequency dependent polarization tensors can be easily obtained from multistatic measurements by solving a linear system while the derivation of the generalized polarization tensors from measurements requires more delicate treatment. The proposed methods are numerically implemented to demonstrate their validity and efficiency.
\end{abstract}

\section{INTRODUCTION}

In this paper we deal with the problem of imaging and characterizing an electromagnetic target using multistatic response (MSR) matrix measurements at a single frequency. The size of the target is supposed to be less than the operating wavelength. Moreover, we assume that we have coincident transmitter and receiver arrays of $N$ elements. The multistatic response (MSR) matrix is the transmitreceive responses of this array.

Our aim in this paper is to exploit the possibility of imaging fine details of the shape of the target and reconstructing its material properties (electric permittivity and magnetic permeability) as well by using the new notion of higher-order frequency-dependent polarization tensors (FDPTs). A remarkable feature of our

Received by the editor August 18, 2010, and, in revised form, December 2, 2010.

2010 Mathematics Subject Classification. Primary 35R30, 35B30, 78M50.

Key words and phrases. Generalized polarization tensors, frequency dependent polarization tensors, asymptotic expansions, shape recovery, target reconstruction, wave imaging. 
method is the fact that these FDPTs can be obtained from the MSR measurements by just solving a linear system.

There have been significant works on imaging small acoustic and electromagnetic targets from the MSR matrix. Ammari et al. 3, 4 proposed a MUSIC (which stands for MUltiple SIgnal Classification) type algorithm based on the asymptotic expansion formula of [21, and successfully reconstructed the location of the target. See also the recent papers 2, 12 for wave imaging of electromagnetic cracks. We emphasize that in the above mentioned works the location and the polarization tensor (PT) are reconstructed. The PT can be interpreted as the zero-frequency limit of the first-order FDPT. The reconstructed PT provides geometric information depicted by the equivalent ellipse or ellipsoid. The purpose of this paper is to extend the method proposed in [9] in order to reconstruct electromagnetic parameters and geometric information finer than the equivalent ellipse or ellipsoid. Compared to the conductivity case in [9], the cost functional here measures the discrepancy between the computed and measured high-order FDPTs rather than between the generalized polarization tensors. The main reason for such a modification of the cost functional is the fact that the (measured) frequency dependent polarization tensors can be, as will be shown later, easily obtained from multistatic measurements by solving a linear system while the derivation of the GPTs from MSR measurements requires more delicate treatment.

To be more precise, let $\epsilon_{0}$ and $\mu_{0}$ be, respectively, the electric permittivity and the magnetic permeability of the free space and let $\epsilon$ and $\mu$ be those of the target. Suppose that the target $D$ is of the form

$$
D=\delta B+z
$$

where $\delta$ is a small parameter representing the characteristic size of the target, $B$ is a reference domain of volume 1 whose center of mass is the origin, and $z$ indicates the location of the target. The problem we consider is to reconstruct $\epsilon, \mu, z$, and some geometric information on $D$ such as its volume $|D|$ and its shape.

Suppose that we have coincident transmitter and receiver arrays, say $\left\{y_{1}, \ldots, y_{N}\right\}$. Suppose also that the distance between the target and the array of transmitters and receivers is much larger than the size of the target. At each point $y_{i}$ of the transmitter the outgoing Green function is generated, and then the scattered wave is recorded at the receiver $y_{j}$ for each $j$, which provides a MSR matrix of size $N \times N$.

The basic idea for reconstructing the target is that the significant singular values of the MSR matrix corresponding to a target of characteristic size less than a wavelength are in fact functions of the high-order FDPTs associated to the target. Indeed, the singular vectors are written in terms of the free space Green function and its high-order derivatives on the array. These fine properties of the MSR matrix allow imaging of fine shape details of the target as well as reliable reconstruction of its physical parameters. In this connection, we refer to [23] for the analysis of the MSR matrix under the Born approximation.

To see this quantitatively, we use the asymptotic formula obtained in [6]. This asymptotic formula for the scattered wave as the size $\delta$ of the target tends to 0 contains higher-order terms and is written in terms of the FDPTs instead of the PTs. This formula clearly reveals that the MSR matrix can be described approximately in terms of the Green function and its derivatives together with higher-order FDPTs. 
To locate the target, we can use exactly the same estimator as in [3, 4, 18, which peaks at the location of the target. This estimator is in fact a MUSIC (which stands for MUltiple SIgnal Classification) imaging functional. For other direct algorithms, we refer, for instance, to 22. Once the target is located, then we can find highorder FDPTs from the MSR matrix by solving a linear system. These FDPTs carry information richer than the usual PTs. We are able to reconstruct electromagnetic parameters from these terms. We also reconstruct geometric information finer than the equivalent ellipse using an optimization procedure. The optimization procedure uses the shape derivative formula of the PTs obtained in [9, which deals with the closely related problem of reconstructing finer details of shape using high-order PTs. Our work here can be seen as an extension of [9] to wave imaging.

As far as we know, it is for the first time that both physical parameters and fine shape details of a target of a characteristic size smaller than the operating wavelength are provided from MSR measurements. For extended targets, we refer the reader to [17, 14, 19, 20, 1.

This paper is organized as follows. In Section 2 we formulate the model problem. In Section 3, we review the asymptotic formula for the scattered wave. For that purpose we discuss the notions of high-order PTs and FDPTs. We also analyze the structure of the MSR matrix using our asymptotic formalism. Section 4 is to present the reconstruction methods for the location, the electromagnetic parameters and fine shape details. Section 5 is devoted to results of numerical simulations. The paper ends with a discussion in Section 6 .

\section{Problem formulation}

Recall that $\mu_{0}>0$ and $\epsilon_{0}>0$ denote the magnetic permeability and electrical permittivity of the free space, respectively, and $\mu>0$ and $\epsilon>0$ those of the target $D$. We assume that $D$ is a bounded $\mathcal{C}^{2}$-domain in $\mathbb{R}^{d}, d=2$ or 3 . We denote by $\nu(x)$ the outward normal to $\partial D$ at $x$.

Let $\omega>0$ denote the frequency and set

$$
k_{0}=\omega \sqrt{\epsilon_{0} \mu_{0}} \quad \text { and } \quad k=\omega \sqrt{\epsilon \mu} .
$$

Let $\Gamma^{k_{0}}(x)$ be the outgoing Green function for $\Delta+k_{0}^{2}$ in $\mathbb{R}^{d}$ corresponding to a Dirac mass at 0 . That is, $\Gamma^{k_{0}}$ is the solution to

$$
\left(\Delta+k_{0}^{2}\right) \Gamma^{k_{0}}(x)=-\delta_{0}(x) \text { in } \mathbb{R}^{d},
$$

subject to the outgoing radiation condition. In three dimensions, the Green function is given by

$$
\Gamma^{k_{0}}(x)=\frac{e^{i k_{0}|x|}}{4 \pi|x|}, \quad x \neq 0
$$

while in two dimensions,

$$
\Gamma^{k_{0}}(x)=\frac{i}{4} H_{0}^{(1)}\left(k_{0}|x|\right), \quad x \neq 0,
$$

where $H_{0}^{(1)}$ is the Hankel function of the first kind of order zero. 
Suppose that $D$ is illuminated by a time-harmonic wave generated at the point source $y$ with the operating frequency $\omega$. In this case, the incident field is given by

$$
U_{y}(x)=\Gamma^{k_{0}}(x, y):=\Gamma^{k_{0}}(x-y),
$$

and the field perturbed in the presence of the target is the solution to the following transmission problem:

(2) $\nabla \cdot\left(\frac{1}{\mu_{0}} \chi\left(\mathbb{R}^{d} \backslash \bar{D}\right)+\frac{1}{\mu} \chi(D)\right) \nabla u_{y}+\omega^{2}\left(\epsilon_{0} \chi\left(\mathbb{R}^{d} \backslash \bar{D}\right)+\epsilon \chi(D)\right) u_{y}=-\frac{1}{\mu_{0}} \delta_{y}$,

and is subject to the outgoing radiation condition, or equivalently,

$$
\begin{cases}\Delta u_{y}+k_{0}^{2} u_{y}=-\delta_{y} & \text { in } \mathbb{R}^{d} \backslash \bar{D} \\ \Delta u_{y}+k^{2} u_{y}=0 & \text { in } D, \\ \left.u_{y}\right|_{+}-\left.u_{y}\right|_{-}=0 & \text { on } \partial D \\ \left.\frac{1}{\mu_{0}} \frac{\partial u_{y}}{\partial \nu}\right|_{+}-\left.\frac{1}{\mu} \frac{\partial u_{y}}{\partial \nu}\right|_{-}=0 & \text { on } \partial D \\ u_{y} \text { satisfies the outgoing radiation condition. } & \end{cases}
$$

Here $\delta_{y}$ is the Dirac mass at $y$ and $\partial / \partial \nu$ denotes the normal derivative on the boundary $\partial D$. Throughout this paper, for a function $u$ defined on $\mathbb{R}^{d} \backslash \partial D$, we denote

$$
\left.\frac{\partial u}{\partial \nu}\right|_{ \pm}(x):=\lim _{t \rightarrow 0^{+}}\langle\nabla u(x \pm t \nu(x)), \nu(x)\rangle, \quad x \in \partial D
$$

if the limits exist. The notation $\left.u\right|_{ \pm}$is understood likewise.

Suppose that we have coincident transmitter and receiver arrays $\left\{y_{1}, \ldots, y_{N}\right\}$ of $N$ elements, used to detect the target. In the presence of the target the scattered field induced on the $i$ th receiving element, $y_{i}$, from the scattering of an incident wave generated at $y_{j}$ can be expressed as follows:

$$
A_{i j}:=\left(u_{y_{j}}-U_{y_{j}}\right)\left(y_{i}\right), \quad i, j=1, \ldots, N .
$$

The problem we consider in this paper is to image the target $D$ from the MSR matrix $A$ of entries $A_{i j}$.

\section{AsYmptotic EXPANSIONS}

Suppose that the anomaly $D$ is parameterized by (11). The purpose of this section is to review the multipolar asymptotic expansions for the scattered field in terms of the reference permittivity, the location, and the shape of the target $D$. This expansion describes the perturbation of the solution caused by the presence of $D$. We begin by reviewing the notions of high-order polarization tensors and frequencydependent polarization tensors. It is worth emphasizing that the regime where our asymptotic results and reconstruction procedures hold is when the characteristic size of the target is smaller than the wavelength $\Lambda:=2 \pi / k_{0}$ and much smaller than the distance $d$ between the target and the array. If the target is of size comparable to the wavelength (say half of the wavelength), then the distance $d$ should be much larger than $\Lambda$. However, if $\delta$ is much smaller than $\Lambda$, then $d$ could be comparable to $\lambda$. 
3.1. High-order PTs and FDPTs. Since $D=\delta B+z$, we consider the following problem after scaling. For a multi-index $\alpha$ with $|\alpha| \geq 0$, let $v_{\alpha}$ be the solution to

$$
\begin{cases}\Delta v+\left(k_{0} \delta\right)^{2} v=0 & \text { in } \mathbb{R}^{d} \backslash \bar{B}, \\ \Delta v+(k \delta)^{2} v=0 & \text { in } B, \\ \left.v\right|_{+}-\left.v\right|_{-}=-x^{\alpha} & \text { on } \partial B, \\ \left.\frac{1}{\mu_{0}} \frac{\partial v}{\partial \nu}\right|_{+}-\left.\frac{1}{\mu} \frac{\partial v}{\partial \nu}\right|_{-}=-\frac{1}{\mu_{0}} \frac{\partial x^{\alpha}}{\partial \nu} & \text { on } \partial B, \\ v \text { satisfies the outgoing radiation condition. } & \end{cases}
$$

Then $v_{\alpha}$ can be represented using the single layer potentials as

$$
v_{\alpha}(x)= \begin{cases}\mathcal{S}_{B}^{k_{0} \delta}\left[\psi_{\alpha}\right](x), & x \in \mathbb{R}^{d} \backslash \bar{B} \\ \mathcal{S}_{B}^{k \delta}\left[\phi_{\alpha}\right](x), & x \in B\end{cases}
$$

for some potentials $\psi_{\alpha}$ and $\phi_{\alpha}$ belonging to $L^{2}(\partial B)$. In fact, they are unique solutions to the system of integral equations

$$
\left\{\begin{aligned}
\mathcal{S}_{B}^{k \delta}\left[\phi_{\alpha}\right]-\mathcal{S}_{B}^{k_{0} \delta}\left[\psi_{\alpha}\right] & =x^{\alpha}, \\
\left.\frac{1}{\mu} \frac{\partial}{\partial \nu} \mathcal{S}_{B}^{k \delta}\left[\phi_{\alpha}\right]\right|_{-}-\left.\frac{1}{\mu_{0}} \frac{\partial}{\partial \nu} \mathcal{S}_{B}^{k_{0} \delta}\left[\psi_{\alpha}\right]\right|_{+} & =\frac{1}{\mu_{0}} \frac{\partial x^{\alpha}}{\partial \nu},
\end{aligned}\right.
$$

The single layer potential $\mathcal{S}_{B}^{k_{0} \delta}$ is defined by

$$
\mathcal{S}_{B}^{k_{0} \delta}[\phi](x):=\int_{\partial B} \Gamma^{k_{0} \delta}(x, y) \phi(y) d \sigma(y), \quad x \in \mathbb{R}^{d} \backslash \bar{B} .
$$

Similarly, we define $\mathcal{S}_{B}^{k \delta}$.

Now let $\Gamma$ be the fundamental solution to $-\Delta$ in $\mathbb{R}^{d}$,

$$
\Gamma(x):= \begin{cases}\frac{1}{4 \pi|x|}, & d=3, \\ -\frac{1}{2 \pi} \log |x|, & d=2,\end{cases}
$$

for $|x| \neq 0$, and let $\mathcal{S}_{B}^{(0)}$ be the single-layer potential associated with $\Gamma$.

Definition 3.1. The (usual) high-order polarization tensor (PT) associated with $B$ and the contrast $\mu_{0} / \mu, M_{\alpha \beta}=M_{\alpha \beta}\left(B, \mu_{0} / \mu\right)$, is defined for multi-indices $\alpha$ and $\beta$ by

$$
M_{\alpha \beta}:=\int_{\partial B} \psi_{\alpha}^{(0)}(w) w^{\beta} d \sigma(w)
$$

where

$$
\left\{\begin{aligned}
\mathcal{S}_{B}^{(0)}\left[\phi_{\alpha}^{(0)}\right]-\mathcal{S}_{B}^{(0)}\left[\psi_{\alpha}^{(0)}\right] & =x^{\alpha}, \\
\left.\frac{1}{\mu} \frac{\partial}{\partial \nu} \mathcal{S}_{B}^{(0)}\left[\phi_{\alpha}^{(0)}\right]\right|_{-}-\left.\frac{1}{\mu_{0}} \frac{\partial}{\partial \nu} \mathcal{S}_{B}^{(0)}\left[\psi_{\alpha}^{(0)}\right]\right|_{+} & =\frac{1}{\mu_{0}} \frac{\partial x^{\alpha}}{\partial \nu}, \quad \text { on } \partial B .
\end{aligned}\right.
$$

The high-order PTs have been extensively studied in [6, 8,

We now introduce the notion of frequency dependent polarization tensors. 
Definition 3.2. With the solution $\left(\phi_{\alpha}, \psi_{\alpha}\right)$ to (7), we define $W_{\alpha \beta}=W_{\alpha \beta}\left(B, \frac{\mu_{0}}{\mu}\right.$, $\left.k_{0} \delta, k \delta\right)$ for multi-indices $\alpha$ and $\beta$ by

$$
W_{\alpha \beta}=\int_{\partial B} \psi_{\alpha}(w) w^{\beta} d \sigma(w) .
$$

We call $W_{\alpha \beta}$ the frequency dependent polarization tensor (FDPT).

For ease of notation, we sometimes use the notation $W_{\alpha \beta}(B)$ when the focus is only on the variation of $B$. It is worth emphasizing that $W_{\alpha \beta}$ depends not only on $B$ but also $k_{0}, k$, and $\delta$ and $\mu_{0} / \mu$.

The following proposition from [6, 7] shows the limiting behavior of $W_{\alpha \beta}$ as $\delta \rightarrow 0$ and makes the connection between $W_{\alpha \beta}$ and $M_{\alpha \beta}$.

Proposition 3.3. The FDPT $W_{\alpha \beta}$ has the following asymptotic behavior as $\delta \rightarrow 0$ : If $|\alpha| \geq 1$ and $|\beta| \geq 1$, then

$$
W_{\alpha \beta}\left(B, \frac{\mu_{0}}{\mu}, k_{0} \delta, k \delta\right) \rightarrow M_{\alpha \beta}\left(B, \frac{\mu_{0}}{\mu}\right) \quad \text { as } \delta \rightarrow 0 .
$$

The proof of Proposition 3.3 is more involved in two dimensions than in three dimensions because of the logarithmic singularity of the Green function [7. Note also that if $|\alpha|=0$ or $|\beta|=0$, then the asymptotics of $W_{\alpha \beta}$ can be found in [6, 7]. For example, in three dimensions, we have

$$
\begin{gathered}
W_{(0,0,0),(0,0,0)}\left(B, \frac{\mu_{0}}{\mu}, k_{0} \delta, k \delta\right)=-\delta^{2} \omega^{2} \epsilon \mu_{0}|B|+O\left(\delta^{3}\right), \\
W_{\alpha,(0,0,0)}\left(B, \frac{\mu_{0}}{\mu}, k_{0} \delta, k \delta\right)=O\left(\delta^{2}\right), \quad|\alpha|=1, \\
W_{(0,0,0), \beta}\left(B, \frac{\mu_{0}}{\mu}, k_{0} \delta, k \delta\right)=O\left(\delta^{2}\right), \quad|\beta|=1 .
\end{gathered}
$$

Similar approximations can be proved in the two-dimensional case. Note that in both the two- and three-dimensional cases, $W_{\alpha \beta}=O\left(\delta^{2}\right)$ for $|\alpha|+|\beta| \leq 1$. On the other hand, the following asymptotic holds in two and three dimensions:

$$
W_{\alpha \beta}\left(B, \frac{\mu_{0}}{\mu}, k_{0} \delta, k \delta\right)=-\delta^{2} \omega^{2} \epsilon_{0} \mu_{0}|B|+O\left(\delta^{4}\right) \quad \text { for }|\alpha|=2 \text { and } \beta=0 .
$$

See [7].

3.2. Multipolar asymptotic expansions. Let $u_{y}$ be the solution to (3) and let $U_{y}$ be the solution in the absence of the target, i.e., $U_{y}(x)=\Gamma^{k_{0}}(x-y)$. Multipolar asymptotic expansions, as $\delta \rightarrow 0$, of the perturbation $u_{y}-U_{y}$ may be described most conveniently using the notion of the FDPTs. In fact, the following multipolar expansion of the perturbation due to the presence of $D=\delta B+z$ was obtained in [6. (12.10)]:

$$
\left(u_{y}-U_{y}\right)(x)=\delta^{d-2} \sum_{p=0}^{n+1} \delta^{p} \sum_{|\alpha|+|\beta|=p} \frac{1}{\alpha ! \beta !} W_{\alpha \beta} \partial_{z}^{\alpha} \Gamma^{k_{0}}(z, y) \partial_{z}^{\beta} \Gamma^{k_{0}}(x, z)+O\left(\delta^{n+d}\right) .
$$

The asymptotic formula (14) holds for $x$ away from $z$. It is a multipolar expansion of the scattered field in the presence of $D$. We emphasize again that $W_{\alpha \beta}$ depends on $\delta$. Indeed, the leading-order term in (14) is of order $\delta^{d}$ since $W_{\alpha \beta}=O\left(\delta^{2}\right)$ for $|\alpha|+|\beta| \leq 1$ and all the higher-order $W_{\alpha \beta}$ are bounded from Proposition 3.3.

As will be shown later, keeping this dependency in $W_{\alpha \beta}$ is convenient from an imaging point of view. We also emphasize that (14) holds not only for $U_{y}(x)=$ 
$\Gamma^{k_{0}}(x, y)$ but also for any solution $U$ to the Helmholtz equation satisfying the radiation condition by replacing $\Gamma^{k_{0}}(z, y)$ on the right-hand side of the equality with $U(z)$.

Combining (91), (10), (11), and (12), together with (13), we can recover from (14) the leading-order term of the scattered field that was obtained in 21] (see also [6. 13]):

$$
\begin{aligned}
u(x)-U(x)= & -\delta^{d}\left[\omega^{2} \mu_{0}\left(\epsilon-\epsilon_{0}\right)|B| U(z) \Gamma^{k_{0}}(x, z)+M \nabla U(z) \cdot \nabla_{z} \Gamma^{k_{0}}(x, z)\right] \\
& +O\left(\delta^{d+1}\right),
\end{aligned}
$$

where $M=\left(M_{\alpha, \beta}\right)_{|\alpha|=|\beta|=1}$ is the polarization tensor (PT) associated with $B$ and the ratio $\mu_{0} / \mu$. Note that (15) is a dipolar approximation.

The asymptotic formulas (91)-(13) show that using $W_{\alpha \beta}$ for $|\alpha|,|\beta|=0,1$, and $W_{(2,0),(0,0)}$, one can approximately reconstruct the volume, the equivalent ellipse, and the electric permittivity of the target. In fact, (13) yields an approximation of $|D|=\delta^{2}|B|$, and then (10) yields $\epsilon$. The formula (9) for $|\alpha|=|\beta|=1$ yields the polarization tensor, and hence the equivalent ellipse of the target. It is timely to mention that the location of $D$ can be reconstructed using the method developed in [3. We may go even further to separate out the information on $\mu_{0} / \mu$ since we have information on both $|D|$ and the polarization tensor. So, this paper mostly concentrates on how to recover $W_{\alpha \beta}$ from the MSR matrix and how to obtain finer details of the target shape and improved information on $\mu_{0} / \mu$ using higher-order $W_{\alpha \beta}$.

3.3. Multistatic response matrix. In this subsection we analyze the structure of the MSR matrix, using the multipolar expansion (14). We first rewrite (14) as follows:

$$
\begin{aligned}
\left(u_{y}-U_{y}\right)(x) & =\delta^{d-2} W_{(0,0),(0,0)} \Gamma^{k_{0}}(z, y) \Gamma^{k_{0}}(x, z) \\
& +\delta^{d-2} \cdot \delta \sum_{|\alpha|+|\beta|=1} W_{\alpha \beta} \partial_{z}^{\alpha} \Gamma^{k_{0}}(z, y) \partial_{z}^{\beta} \Gamma^{k_{0}}(x, z) \\
& +\delta^{d-2} \sum_{p=2}^{n+1} \delta^{p} \sum_{|\alpha|+|\beta|=p} \frac{1}{\alpha ! \beta !} W_{\alpha \beta} \partial_{z}^{\alpha} \Gamma^{k_{0}}(z, y) \partial_{z}^{\beta} \Gamma^{k_{0}}(x, z) \\
& +O\left(\delta^{n+d}\right) .
\end{aligned}
$$

The first two terms in (16) are the sum of point source and dipolar approximations of the target while the third term gives a multipolar approximation of the target written in terms of higher-order derivatives of the Green function.

Recall now that we have coincident transmitter and receiver arrays $\left\{y_{1}, \ldots, y_{N}\right\}$ of $N$ elements, used to detect the target located at $z$. In the presence of the target the scattered field induced on the $i$-th receiving element from the scattering of an incident wave generated at $y_{j}$ can be approximated using the multipolar expansion (16). The following proposition holds.

Proposition 3.4. We have

$$
\left(u_{y_{j}}-U_{y_{j}}\right)\left(y_{i}\right)=\mathcal{G}\left(y_{i}, z\right) \mathcal{W G}\left(y_{j}, z\right)^{T}+O\left(\delta^{n+d}\right),
$$


where $T$ denotes the transpose, $\mathcal{G}\left(y_{i}, z\right)$ is a row vector of size $\frac{1}{2}(n+1)(n+2)$ in dimension two and $\frac{1}{6}(n+1)(n+2)(n+3)$ in the three-dimensional case, which is given by

$$
\mathcal{G}\left(y_{i}, z\right)=\left(\frac{1}{\alpha !} \partial_{z}^{\alpha} \Gamma^{k_{0}}\left(y_{i}, z\right)\right)_{|\alpha| \leq n}
$$

and $\mathcal{W}$ is defined by

$$
\mathcal{W}=\left(\mathcal{W}_{\alpha \beta}\right)_{|\alpha|,|\beta| \leq n}=\left(\delta^{d-2+|\alpha|+|\beta|} W_{\alpha \beta}\right)_{|\alpha|,|\beta| \leq n} .
$$

If $\delta$ is small, then higher-order terms can be neglected. In this case, the analysis of the MSR matrix reduces to the classical one which is based on a dipolar approximation. As $\delta$ is increasing, more and more multipolar terms should be included in formula (16) in order to well approximate the response of the target. We also emphasize that in the approximation (17) there are some terms which do not appear in (16). But these terms are all of order $O\left(\delta^{n+d}\right)$, and hence do not play a role in the approximation.

In view of (18), the signal space of the MSR matrix becomes richer. The set of singular vectors consists of the Green function and its high derivatives on the array. Then, in order to locate the target, exactly the same estimator constructed in [3, 4] can be used. This estimator peaks at the location of the target. However, the significant singular values are perturbed, even those associated to the dipolar approximation. The difference between those based on a point approximation and those based on a multipolar approximation (high-order approximation) are measured in terms of the difference between the polarization tensor $M$ and the new quantities $W_{\alpha \beta}$ for $|\alpha|,|\beta|=0,1$. Indeed, when $\delta$ is increasing, new significant singular values can emerge. Those are related to higher-order multipolar terms. They can be expressed in terms of $W_{\alpha \beta}$ for $|\alpha|$ or $|\beta| \geq 2$. These new singular values, which are intermediate between the larger ones and zero, contain some information on the target and give better approximation of its shape and electromagnetic parameters. In this connection, see [15, 1.

\section{Reconstruction procedures}

In this section, we focus on the two-dimensional case. Our procedures can be in principle extended to three dimensions.

It is known that the polarization tensor contains a mixture of geometric information and material parameters $[6$. It is then impossible to reconstruct separately the volume and the material parameters of a target from the dipolar approximation. In other terms, it is impossible to extract such information from the first significant singular values of the MSR matrix.

However, as we have seen in subsection 3.2, if we recover high-order FDPTs, $W_{\alpha \beta}$, we are able to determine the volume of the target, and hence it is possible to separate the information on the material property from geometric information. In this section we present reconstruction procedures. We begin by recalling how to reconstruct the location of the target. We then determine the electromagnetic parameters and the equivalent ellipse. The final procedure adapts an optimal control approach to obtain shape details of the target that are finer than its equivalent ellipse. 
4.1. Location. Location search is based on a MUSIC-type algorithm which has been used in various contexts; see, for example, [3, 12, 2, 10.

Let $A=\left(A_{i j}\right)$ denote the MSR matrix defined by (4). We compute the singular value decomposition (SVD) of $A$. Let $\lambda_{1} \geq \lambda_{2} \geq \cdots$ be the singular values of $A$, and let $u_{p}$ and $v_{p}$ be the corresponding left and right singular vectors, respectively. Then $A$ has the spectral decomposition

$$
A=\sum \lambda_{p} u_{p} \otimes v_{p}
$$

Using the first $m$ singular vectors, we define an orthogonal projector $P_{m}$ on $\operatorname{span}\left\{u_{1}, \cdots, u_{m}\right\}$ as $P_{m}=\sum_{p=1}^{m} u_{p} \otimes u_{p}$. We then define a minimization functional $L_{m}(z)$ for $z$ in the search domain $\Omega$ by

$$
L_{m}(z):=\frac{\sum_{|\alpha| \leq 1}\left\|\left(I-P_{m}\right)\left(\partial_{z}^{\alpha} \Gamma^{k_{0}}(\cdot, z)\right)\right\|_{L^{2}(\partial \Omega)}}{\sum_{|\alpha| \leq 1}\left\|P_{m}\left(\partial_{z}^{\alpha} \Gamma^{k_{0}}(\cdot, z)\right)\right\|_{L^{2}(\partial \Omega)}} .
$$

We try to find a minimizer $z^{\star}$ of the functional,

$$
L_{m}\left(z^{\star}\right)=\min _{z \in \Omega} L_{m}(z)
$$

In the simulations, $m=6$ seems to be an adequate value.

4.2. Size and permittivity. Once the location of the target is recovered, the matrix $\mathcal{W}$ in (19) can be recovered from the MSR matrix $A$. In fact, if we denote by $z^{\star}$ the recovered location of the target, then we can find $\mathcal{W}$ by solving

$$
A_{i j} \approx \mathcal{G}\left(y_{i}, z^{\star}\right) \mathcal{W G}\left(y_{j}, z^{\star}\right)^{T},
$$

where $\mathcal{G}\left(y_{i}, z^{\star}\right)$ is given by (18). This is a system of $N \times N$ linear equations with $\left[\frac{1}{2}(n+1)(n+2)\right]^{2}$ (the number of entries of $\mathcal{W}$ ) unknowns in two dimensions. Hence it can be solved in general if we take $N$ sufficiently large. We solve (22) in the minimum residual sense. In actual simulation we only use an $N \times m$ submatrix of $A$ where $m=\frac{1}{2}(n+1)(n+2)$, which amounts to using $N$ transmitters and $m$ receivers.

In view of (10) and (13), the size $|D|$ and the electrical permittivity $\epsilon$ of the target can be estimated as follows:

$$
|D|=\frac{1}{\omega^{2} \epsilon_{0} \mu_{0}}\left|W_{(2,0),(0,0)}\right|
$$

and

$$
\epsilon=\frac{\left|W_{(0,0),(0,0)}\right|}{\mu_{0} \omega^{2}|D|} .
$$

We emphasize that the estimations in (23) and (24) hold regardless of the shape of the target since the leading-order terms in (10) and (13) are shape independent.

4.3. Equivalent ellipse and permeability. We now describe a procedure to obtain the equivalent ellipse representing the shape of the target using $W_{\alpha \beta}$ for $|\alpha|=|\beta|=1$. To this end we need to review the explicit form of the polarization tensor $M(E, \kappa)=\left(M_{i j}\right)$ associated with an ellipse $E$ and material contrast $\kappa$. We also review a method to recover the ellipse and $\kappa$ from its PT (and the size). 
See [16] or [6] for the details. Here we use the identifications $M_{11}=M_{(0,1),(0,1)}$, $M_{12}=M_{(0,1),(1,0)}$, etc.

Let $E^{\prime}$ be an ellipse with semi-major axis of length $a$ and semi-minor axis of length $b$, whose focal line is on either the $x_{1}$ - or the $x_{2}$-axis, and let $E=R E^{\prime}$ where $R=\left(\begin{array}{cc}\cos \theta & -\sin \theta \\ \sin \theta & \cos \theta\end{array}\right)$, the rotation by the angle $\theta$. Let $M$ and $M^{\prime}$ be the polarization tensors of $E$ and $E^{\prime}$, respectively. Then $M^{\prime}$ takes the form

$$
M^{\prime}=(\kappa-1)\left|E^{\prime}\right|\left(\begin{array}{cc}
\frac{a+b}{a+\kappa b} & 0 \\
0 & \frac{a+b}{b+\kappa a}
\end{array}\right) \text {, }
$$

and $M$ is given by

$$
M=R M^{\prime} R^{T}
$$

In view of (25) and (26), the parameters $\theta$ and $\kappa$ can be recovered as follows:

$$
\begin{gathered}
\frac{M_{12}+M_{21}}{M_{22}-M_{11}} \tan 2 \theta, \quad-\frac{\pi}{4}<\theta \leq \frac{\pi}{4}, \\
\kappa=\frac{|E|\left(M_{11}^{\prime}+M_{22}^{\prime}\right)+M_{22}^{\prime} M_{11}^{\prime}}{M_{22}^{\prime} M_{11}^{\prime}-|D|\left(M_{22}^{\prime}+M_{11}^{\prime}\right)},
\end{gathered}
$$

and for $a$ and $b$, one should solve

$$
\frac{a}{b}=\frac{M_{22}^{\prime}-M_{11}^{\prime} \kappa}{M_{11}^{\prime}-M_{22}^{\prime} \kappa} \quad \text { and } \quad a b \pi=|E| .
$$

Let

$$
\mathcal{W}_{(1)}=\left(\mathcal{W}_{i j}\right)_{i, j=1,2}:=\left(\mathcal{W}_{\alpha \beta}\right)_{|\alpha|=|\beta|=1}
$$

Then by (9) we have

$$
\mathcal{W}_{(1)} \approx \delta^{2} M\left(B, \frac{\mu_{0}}{\mu}\right)=M\left(D, \frac{\mu_{0}}{\mu}\right) .
$$

Taking formulas (27), (28), and (29) into account, we may recover $\theta, \kappa$, and $a, b$ from $\mathcal{W}_{(1)}$ in the following manner.

\section{[Equivalent Ellipse Reconstruction Procedure]}

Step 1. If both $\left|\mathcal{W}_{11}-\mathcal{W}_{22}\right|$ and $\left|\mathcal{W}_{21}+\mathcal{W}_{12}\right|$ are smaller than a given tolerance $\tau$, then we assume that the target is a disk.

Step 2. Determine the angle of rotation $\theta$ by solving

$$
\frac{\mathcal{W}_{12}+\mathcal{W}_{21}}{\mathcal{W}_{22}-\mathcal{W}_{11}} \tan 2 \theta, \quad-\frac{\pi}{4}<\theta \leq \frac{\pi}{4}
$$

Step 3. Using the angle $\theta$, find $\mathcal{W}_{(1)}^{\prime}$ by solving,

$$
\mathcal{W}_{(1)}^{\prime}=R(-\theta) \mathcal{W}_{(1)} R(-\theta)^{T}
$$

where $R(-\theta)$ is the rotation by $-\theta$.

Step 4. Using $|D|$ found in (23), compute $\mu$ by

$$
\frac{\mu_{0}}{\mu}=\frac{|D|\left(\mathcal{W}_{11}^{\prime}+\mathcal{W}_{22}^{\prime}\right)+\mathcal{W}_{22}^{\prime} \mathcal{W}_{11}^{\prime}}{\mathcal{W}_{22}^{\prime} \mathcal{W}_{11}^{\prime}-|D|\left(\mathcal{W}_{22}^{\prime}+M_{11}^{\prime}\right)}
$$


Step 5. Compute the lengths of semi-major and minor axis, $a$ and $b$, by solving

$$
\frac{a}{b}=\frac{\mathcal{W}_{22}^{\prime}-\mathcal{W}_{11}^{\prime} \frac{\mu_{0}}{\mu}}{\mathcal{W}_{11}^{\prime}-\mathcal{W}_{22}^{\prime} \frac{\mu_{0}}{\mu}} \quad \text { and } \quad a b \pi=|D| .
$$

The equivalent ellipse and estimated permeability in this subsection already provide good information on the shape and permeability of the target. The equivalent ellipse will play the role of a good initial guess for the iterative scheme in the next subsection to find finer geometric features of shape using higher-order $\mathcal{W}_{\alpha \beta}$.

It is worth emphasizing that we are able to separate the permeability contrast $\mu_{0} / \mu$ from $|D|$ thanks to the extra information provided by $\mathcal{W}_{(2,0),(0,0)}$. It is wellknown that it is impossible to separate $\mu_{0} / \mu$ from $|D|$ by only using $\left(\mathcal{W}_{\alpha \beta}\right)_{|\alpha|=|\beta|=1}$. See [6].

4.4. Fine shape details. So far we reconstructed the electromagnetic parameters $\epsilon, \mu$, and the equivalent ellipse. We now reconstruct fine details of the shape of the target using higher-order FDPTs.

Suppose that we have the measured $\mathcal{W}^{\text {meas }}=\left(\mathcal{W}_{\alpha \beta}^{\text {meas }}\right)$ at our disposal. Let $W_{\alpha \beta}(D)$ be the FDPT associated with the domain $D$ while the electromagnetic parameters $\epsilon$ and $\mu$ are fixed. Then, by minimizing over $D$ a discrepancy functional between $W_{\alpha \beta}(D)$ and $\mathcal{W}_{\alpha \beta}^{\text {meas }}$ for $|\alpha|+|\beta| \leq K$, we can reconstruct fine details of the shape of the target, which we denote by $D_{\text {target }}$. It is worth mentioning that

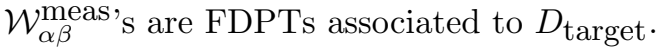

In order to minimize the discrepancy functional we should compute the shape derivative of $W_{\alpha \beta}(D)$. Instead of doing this, we use the asymptotic formula for the PT $M_{\alpha \beta}(D)$ under small deformations of the shape of $D$.

Let $D$ be a bounded domain and $D_{\eta}$ be an $\eta$-perturbation of $D$, where $\eta$ is a small parameter, such that

$$
\partial D_{\eta}:=\left\{x_{\eta}=x+\eta h(x) \nu(x) \mid x \in \partial D, h \in \mathcal{C}^{1}(\partial D)\right\},
$$

where $\nu$ is the unit outward normal to $\partial D$.

Let us now suppose that $a_{\alpha}$ and $b_{\beta}$ are constants such that $H(x):=\sum_{\alpha} a_{\alpha} x^{\alpha}$ and $F(x):=\sum_{\beta} b_{\beta} x^{\beta}$ are harmonic polynomials. Let $\kappa=\mu_{0} / \mu$ and let $u$ and $v$ be, respectively, the solutions to

$$
\begin{cases}\Delta u=0, & \text { in } D \cup\left(\mathbb{R}^{2} \backslash \bar{D}\right), \\ \left.u\right|_{+}-\left.u\right|_{-}=0, & \text { on } \partial D, \\ \left.\frac{\partial u}{\partial \nu}\right|_{+}-\left.\kappa \frac{\partial u}{\partial \nu}\right|_{-}=0, & \text { on } \partial D, \\ (u-H)(x)=O\left(|x|^{-1}\right) & \text { as }|x| \rightarrow \infty\end{cases}
$$

and

$$
\begin{cases}\Delta v=0, & \text { in } D \cup\left(\mathbb{R}^{2} \backslash \bar{D}\right), \\ \left.\kappa v\right|_{+}-\left.v\right|_{-}=0, & \text { on } \partial D, \\ \left.\frac{\partial v}{\partial \nu}\right|_{+}-\left.\frac{\partial v}{\partial \nu}\right|_{-}=0, & \text { on } \partial D, \\ (v-F)(x)=O\left(|x|^{-1}\right) & \text { as }|x| \rightarrow \infty .\end{cases}
$$

The following asymptotic formula was obtained in 9 . 
Proposition 4.1. We have

$$
\begin{aligned}
& \sum_{\alpha, \beta} a_{\alpha} b_{\beta} M_{\alpha \beta}\left(D_{\eta}, \kappa\right)-\sum_{\alpha, \beta} a_{\alpha} b_{\beta} M_{\alpha \beta}(D, \kappa) \\
& =\eta(\kappa-1) \int_{\partial D} h(x)\left[\left.\left.\frac{\partial v}{\partial \nu}\right|_{-} \frac{\partial u}{\partial \nu}\right|_{-}+\left.\left.\frac{1}{k} \frac{\partial u}{\partial T}\right|_{-} \frac{\partial v}{\partial T}\right|_{-}\right]_{(x) d \sigma(x)+O\left(\eta^{2}\right) .}
\end{aligned}
$$

In view of (9), we now have the following approximation formula which holds for $\eta$ much smaller than $\delta$.

Corollary 4.2. For $\eta \ll \delta$, we have

$$
\begin{aligned}
& \sum_{\alpha, \beta} a_{\alpha} b_{\beta} W_{\alpha \beta}\left(D_{\eta}\right)-\sum_{\alpha, \beta} a_{\alpha} b_{\beta} W_{\alpha \beta}(D) \\
& \approx \eta\left(\frac{\mu_{0}}{\mu}-1\right) \int_{\partial D} h(x)\left[\left.\left.\frac{\partial v}{\partial \nu}\right|_{-} \frac{\partial u}{\partial \nu}\right|_{-}+\left.\left.\frac{1}{k} \frac{\partial u}{\partial T}\right|_{-} \frac{\partial v}{\partial T}\right|_{-}\right]_{(x) d \sigma(x) .}
\end{aligned}
$$

We now define the functional $J_{\epsilon, \mu}$ to be minimized by

$$
J_{\epsilon, \mu}(D):=\sum_{1 \leq|\alpha|+|\beta| \leq K}\left|\sum_{\alpha, \beta} a_{\alpha} b_{\beta} W_{\alpha \beta}(D)-\sum_{\alpha, \beta} a_{\alpha} b_{\beta} \mathcal{W}_{\alpha \beta}^{\text {meas }}\right|^{2},
$$

where the first sum is over various $a_{\alpha}$ and $b_{\beta}$ such that $H=\sum a_{\alpha} x^{\alpha}$ and $F=$ $\sum b_{\beta} x^{\beta}$ are homogeneous harmonic polynomials. Let

$$
\phi_{H F}(x)=(k-1)\left[\left.\left.\frac{\partial v}{\partial \nu}\right|_{-} \frac{\partial u}{\partial \nu}\right|_{-}+\left.\left.\frac{1}{k} \frac{\partial u}{\partial T}\right|_{-} \frac{\partial v}{\partial T}\right|_{-}\right],
$$

where $u$ and $v$ are solutions to (35) and (36), respectively. The approximate formula (38) shows the following.

Proposition 4.3. The shape derivative of $J_{\epsilon, \mu}(D)$ is approximately given by

$$
\left\langle d_{S} J_{\epsilon, \mu}(D), h\right\rangle_{L^{2}(\partial D)} \approx \sum_{1 \leq|\alpha|+|\beta| \leq K} \delta_{H F}\left\langle\phi_{H F}, h\right\rangle_{L^{2}(\partial D)},
$$

where

$$
\delta_{H F}=\sum_{\alpha, \beta} a_{\alpha} b_{\beta} W_{\alpha \beta}(D)-\sum_{\alpha, \beta} a_{\alpha} b_{\beta} \mathcal{W}_{\alpha \beta}^{\text {meas }} .
$$

As in [9], we can make the optimization procedure recursively by increasing $K$ to refine the shape reconstruction. At each step, the initial guess for the shape is the result of the previous one. The equivalent ellipse in subsection 4.3 provides a good initial guess to start with.

\section{[Generalization of the Recursive Shape Reconstruction Procedure of [9]}

Step 1. As an initial guess, choose $\epsilon_{e}, \mu_{e}$ and $D_{e}$ that we reconstruct in the equivalent ellipse reconstruction procedure.

Step 2. Start with $K=3$ and minimize $J_{\epsilon, \mu}(D)$ using the expression (40) of its shape derivative $d_{S} J_{\epsilon, \mu}(D)$. Use the equivalent ellipse as an initial guess.

Step 3. Increase $K$ and find $D_{\text {updated }}$ using the result $D_{\text {given }}$ of the previous step as an initial guess. 
Step 4. Repeat Step 3, until one of two stop criterions holds,

$$
\begin{aligned}
\|h\|_{\partial D_{\text {given }}} & \leq h_{\text {tol }}, \\
\left\|W_{\alpha \beta}\left(D_{\text {given }}\right)-\mathcal{W}_{\alpha \beta}^{\text {meas }}\right\|_{K} & \leq\left\|W_{\alpha \beta}\left(D_{\text {updated }}\right)-\mathcal{W}_{\alpha \beta}^{\text {meas }}\right\|_{K}
\end{aligned}
$$

where

$$
\left\|W_{\alpha \beta}\right\|_{K}:=\sum_{1 \leq|\alpha|+|\beta| \leq K}\left|\sum_{\alpha, \beta} a_{\alpha} b_{\beta} W_{\alpha \beta}\right|^{2} .
$$

However, in this paper we propose a new recursive optimization algorithm. Instead of making the recursion on the order $K$ of the used FDPTs, we fix, in Step 3, $K$ equal to the maximum of $|\alpha|+|\beta|$ corresponding to the measured $\mathcal{W}_{\alpha \beta}^{\text {meas }}$. We set $D_{\text {given }}$ to be the initial guess and $\eta$ to be a fixed small parameter. Assuming that the target is a small deformation of the reconstructed initial guess, we can reduce by using (37) the minimization of $J_{\epsilon, \mu}(D)$ to that of (recursively) minimizing over $h$ the functional

$$
\begin{aligned}
J^{(K)}(h) & :=\sum_{1 \leq|\alpha|,|\beta| \leq K} \mid \sum_{\alpha, \beta} a_{\alpha} b_{\beta} W_{\alpha \beta}\left(D_{\text {given }}\right)-\sum_{\alpha, \beta} a_{\alpha} b_{\beta} \mathcal{W}_{\alpha \beta}^{\text {meas }} \\
& -\left.\eta(\kappa-1) \int_{\partial D_{\text {given }}} h(x)\left[\left.\left.\frac{\partial v}{\partial \nu}\right|_{-} \frac{\partial u}{\partial \nu}\right|_{-}+\left.\left.\frac{1}{k} \frac{\partial u}{\partial T}\right|_{-} \frac{\partial v}{\partial T}\right|_{-}\right](x) d \sigma(x)\right|^{2},
\end{aligned}
$$

where $h$ belongs to classes of more and more oscillating basis functions on $\partial D_{\text {given }}$.

\section{NumericAl EXPERIMENTS}

We now present the results of numerical experiments to validate the method proposed in this paper. The results are of two kinds: one for the reconstruction of equivalent ellipses and electromagnetic parameters, and the second for the optimization procedure to reconstruct fine shape details. We begin with a few words on the forward solver.

5.1. Convergence of the forward solver. In order to collect the data, we solve the direct problem (3) using the single layer potential as follows:

$$
u(x)=\left\{\begin{array}{l}
U(x)+\mathcal{S}_{D}^{k_{0}}[\psi](x), \quad x \in \mathbb{R}^{2} \backslash \bar{D}, \\
\mathcal{S}_{D}^{k}[\varphi](x), \quad x \in D
\end{array}\right.
$$

where $\psi$ and $\varphi$ satisfy the following relations:

$$
\left\{\begin{aligned}
\mathcal{S}_{D}^{k}[\varphi]-\mathcal{S}_{D}^{k_{0}}[\psi] & =U, \\
\left.\frac{1}{\mu} \frac{\partial}{\partial \nu} \mathcal{S}_{D}^{k}[\varphi]\right|_{-}-\left.\frac{1}{\mu_{0}} \frac{\partial}{\partial \nu} \mathcal{S}_{D}^{k_{0}}[\psi]\right|_{+} & =\frac{1}{\mu_{0}} \frac{\partial U}{\partial \nu}, \quad \text { on } \partial D .
\end{aligned}\right.
$$

A generalized minimum residual (GMRES) method has been used to solve the system of the integral equations (45) and the single layer potential has been numerically evaluated using the high-order numerical quadrature rule with $M$ equally spaced discretization points along $\partial \Omega$ and $\partial D$. Here $\Omega$ is a domain on the boundary of which the transducers are located. In other words, we collect the MSR matrix on $\partial \Omega$.

In all the numerical experiments in this section, we assume that $\Omega=]-1,1[\times]-$ $1,1[$ and the operating frequency $\omega=0.1$.

In Figure 1 the inclusion $D$ is with semi-axes lengths 0.1 and 0.05 and angle of orientation $\theta=25$ degrees. The electromagnetic parameters are given by 

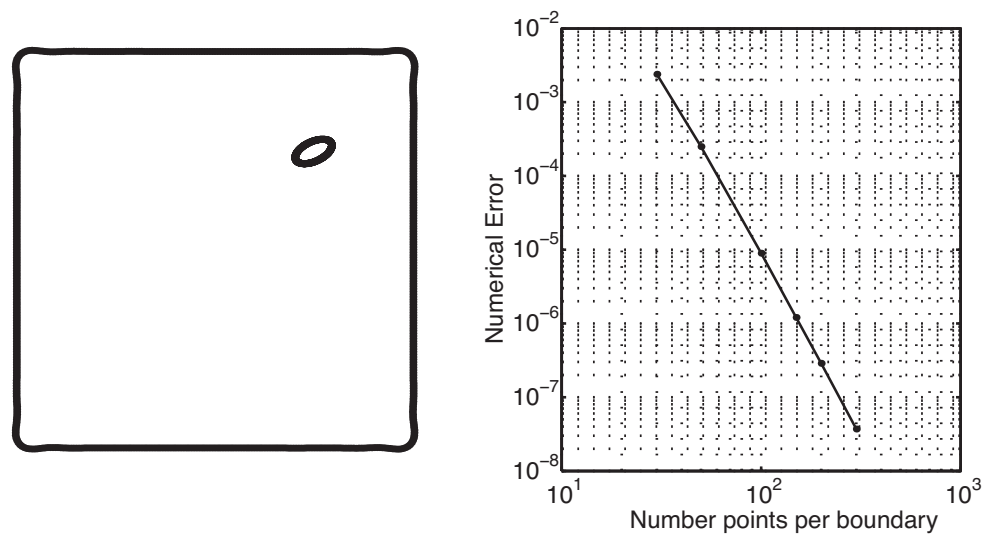

FiguRE 1. Convergence error of the forward solver with 30-300 discretization points for the domain with ellipse-shaped target. The solid line represents the relative convergence error of $(u-$ $U)\left.\right|_{\partial \Omega}$.

$\left(\mu_{0}, \epsilon_{0}\right)=(4,1),(\mu, \epsilon)=(121,25)$. Note that throughout this section all the angles are measured in degrees. Figure 1 shows the relative convergence error of the forward solver for the computation of $(u-U)(x)$ on $x \in \partial \Omega$. We compute coarse grid solutions with $M=30-300$ equi-spaced points on $\partial \Omega$ and compare them with the corresponding forward solution on the finer grid with $M=600$.

In the reconstruction procedures, we set the order of accuracy of the direct code to be fifth in order to achieve around six digits of accuracy with $M=160$.

5.2. Accuracy of the computed polarization tensor. In this subsection, we check the computational accuracy of the $\mathcal{W}$, which is reconstructed from the MSR matrix by solving (22). It is worth mentioning that we do not use full MSR matrix. We only use its submatrix to be able to solve (22). We compare $\mathcal{W}$ (obtained from the MSR matrix) with the (exact) tensor $\mathcal{W}^{\mathrm{d}}$ given by (8) for the numerically computed forward solution of (45). The target is of elliptic shape. Figure 2 shows the relative difference,

$$
\frac{\sum_{|\alpha|,|\beta| \leq 2}\left|\mathcal{W}_{\alpha \beta}-\mathcal{W}_{\alpha \beta}^{\mathrm{d}}\right|^{2}}{\sum_{|\alpha|,|\beta| \leq 2}\left|\mathcal{W}_{\alpha \beta}^{\mathrm{d}}\right|^{2}}
$$

as a function of the size of the ellipse.

The larger the size of the target, the bigger the difference, which is quite natural. The straight line on the right-hand-side of the log-log plot indicates that the asymptotic truncation error is about second-order, $O\left(\delta^{2}\right)$. The error gets larger again when the target becomes extremely small, say $\delta<10^{-3}$. It is caused by the forward numerical solver which could not provide adequate precision with given number of discretization points. We set the discretization points $M=160$ for our simulations, which gives about 3 digits of accuracy for $\mathcal{W}$ in the range of $10^{-4}<\delta<10^{-1}$. 

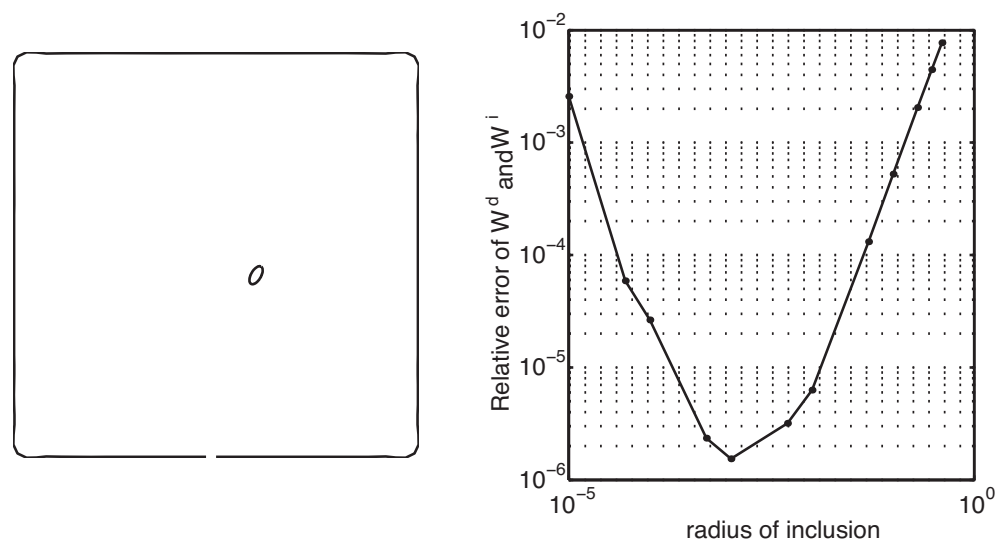

Figure 2. The comparison of $\mathcal{W}$ and $\mathcal{W}^{\mathrm{d}}$. The plots show the relative difference for an ellipse-shaped target.

5.3. Electromagnetic parameters and the equivalent ellipse. In this subsection, we present computational results for reconstruction of electromagnetic parameters and the equivalent ellipses of targets of various shape using the methods proposed in Subsections 4.2 and 4.3. We emphasize that the electromagnetic parameters, $\mu$ and $\epsilon$, are unknown in advance. First, Figure 3 shows the reconstructed equivalent ellipses and $\mu$ and $\epsilon$ for a single target of elliptic shape.

Figure 4 summarizes the computed results for a target of general shape. It demonstrates that the reconstruction algorithm works pretty well even for targets of general shapes.

5.4. Fine shape details. In this subsection we test the optimization algorithm in Subsection 4.4 to find fine details of the shape of the target. We set $\mu$ and $\epsilon$ to be the values found by the equivalent ellipse reconstruction method, and use the equivalent ellipse as an initial guess. With them, we find a better approximation of the shape of the target.

For reconstructing the shape deformation, we take $h$ of the form

$$
h(\theta):=a_{0}+\sum_{\ell=1}^{s}\left(a_{\ell}^{c} \cos (\ell \theta)+a_{\ell}^{s} \sin (\ell \theta)\right),
$$

where $a_{\ell}^{c}$ and $a_{\ell}^{s}$ are constants to be found by minimizing (43). In the following examples, we refine the domain by increasing the order $s$ of $h$ while fixing the order $K$ of the FDPT $W_{\alpha \beta}$ to be $7,|\alpha|+|\beta| \leq K=7$. Then Step 2 in subsection 4.4 becomes $s=1, K=7$ and Step 3 becomes $s=s+1, K=7$.

Figure 5 shows the computational results of a single inclusion with $(\mu, \epsilon)=$ $(36,25)$ in the homogeneous background $\left(\mu_{0}, \epsilon_{0}\right)=(4,1)$ material. The leftmost figures show an initial guess from ellipse reconstruction algorithm which gives initial shape and material parameter $\left(\mu_{e}, \epsilon_{e}\right)=(35.3163,25.1867)$. The remaining figures show the result when $s$ becomes 3,5 , and 6 . The algorithm stops at the step $s=5$ since the difference of $W_{\alpha, \beta}\left(D_{\text {updated }}\right)-\mathcal{W}_{\alpha, \beta}^{\text {meas }}$ for $s=6$ is larger than that for $s=5$. 

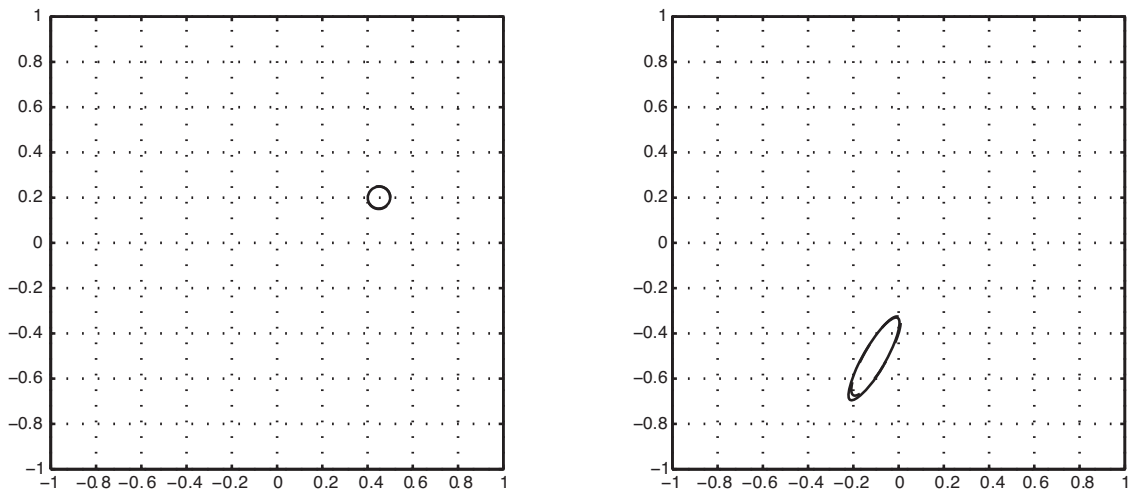

\begin{tabular}{|c|c|c|}
\hline & actual data & computed data \\
\hline$z$ & $(0.4500,0.2000)$ & $(0.4508,0.2005)$ \\
$\delta$ & 0.0500 & $0.0488,0.0498$ \\
$\theta$ & - & -32.4909 \\
$\mu$ & 64 & 80.2815 \\
$\epsilon$ & 25 & 25.9793 \\
\hline & actual data & computed data \\
\hline$z$ & $(-0.1000,-0.5000)$ & $(-0.1086,-0.5126)$ \\
$\delta$ & $0.2000,0.0500$ & $0.2089,0.0500$ \\
$\theta$ & 60 & 60.1418 \\
$\mu$ & 121 & 102.2785 \\
$\epsilon$ & 36 & 32.1234 \\
\hline
\end{tabular}

FiguRE 3. Reconstructed ellipses for disk or ellipse shaped targets marked with dashed-dotted curves. The solid ellipse are reconstructed equivalent ellipses. The two values of computed delta are the axes lengths of the reconstructed ellipse. We take $\left(\mu_{0}, \epsilon_{0}\right)=(4,1),(9,6)$ in the first and second plots, respectively.

Figure 6 again shows the computational results of a single star-shaped inclusion with $(\mu, \epsilon)=(121,25)$ in the background $\left(\mu_{0}, \epsilon_{0}\right)=(4,1)$ material. The initial ellipse shape with $\left(\mu_{e}, \epsilon_{e}\right)=(122.8574,25.5167)$ is given in the leftmost figures. The remaining figures show the result when $s$ becomes 3,4 , and 5 . The algorithm stops at step $s=4$ since the updated boundary distance $h$ is pretty small and no further updates are made after $s>4$.

Figure 7 shows the final reconstructed results for three different targets of various shapes. We emphasize that we fixed $K$ to be 7 and increase the order $s$ of $h$ from 1 to $s_{\text {stop }}$ until we reach a termination condition (Step 4 of the reconstruction algorithm in subsection 4.4).

Figure 8 shows the computational results for multiple inclusions with $(\mu, \epsilon)=$ $(36,25)$ and $\left(\mu_{0}, \epsilon_{0}\right)=(4,1)$. The initial guess is the equivalent ellipse with $\left(\mu_{e}, \epsilon_{e}\right)=(36.2370,25.0150)$ and $\left(\mu_{e}, \epsilon_{e}\right)=(34.4308,24.9802)$.

The example in Figure 9 demonstrates the stability of our reconstruction procedure in the case of a moderate noise. The configuration and the material parameters 
are exactly as in Figure 5. However, as shown in Figure 10, the most resolved reconstructions become instable as the amount of noise increases. There is a trade-off between the resolution and the stability.
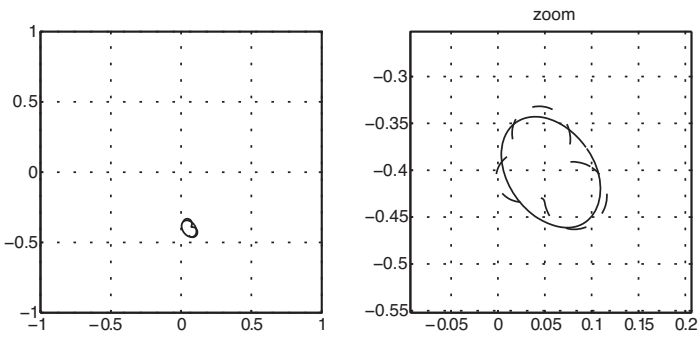

\begin{tabular}{|c|c|c|}
\hline & actual data & computed data \\
\hline$z$ & $(0.0567,-0.4009)$ & $(0.0565,-0.4020)$ \\
$\delta$ & - & $0.0458,0.0648$ \\
$\theta$ & - & 35.2369 \\
$\mu$ & 36 & 48.3027 \\
$\epsilon$ & 25 & 25.0300 \\
\hline
\end{tabular}

FIGURE 4. Reconstructed ellipses for a non-ellipse shaped target marked with dashed-dotted curves. The solid ellipse are reconstructed equivalent ellipse. We take $\left(\mu_{0}, \epsilon_{0}\right)=(4,1)$.
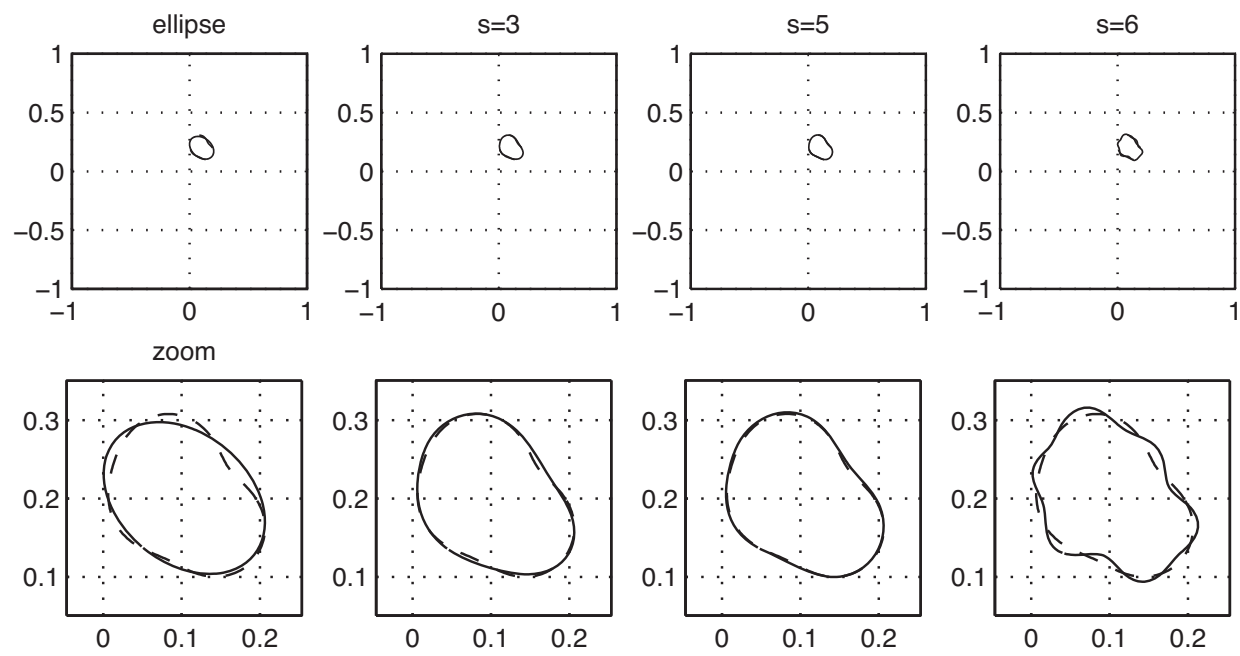

FiguRE 5. Computed shape for a general shaped target marked with dashed-dotted curves. The solid lines are computed increasing the order $s$ of $h$. Bottom figures are 6.67 times zoom-ups of the corresponding results. 

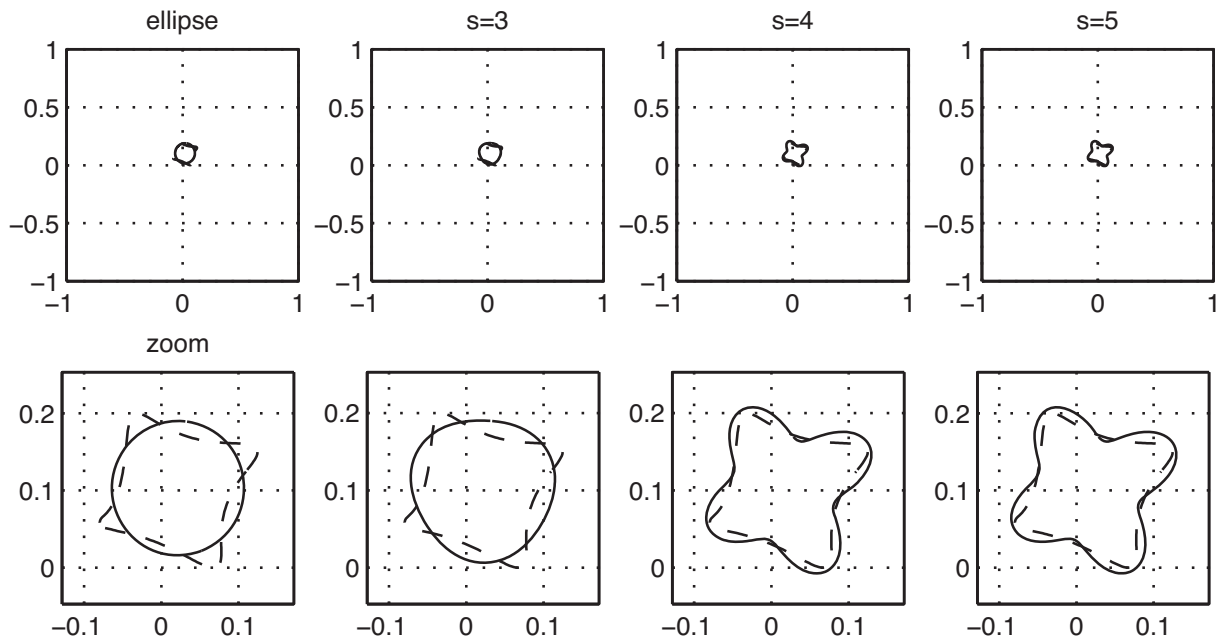

FiguRE 6. Computed shape for a second general shaped target marked with dashed-dotted curves. The solid lines are computed increasing the order $s$ of $h$. Bottom figures are 6.67 times zoom-ups of the corresponding results.
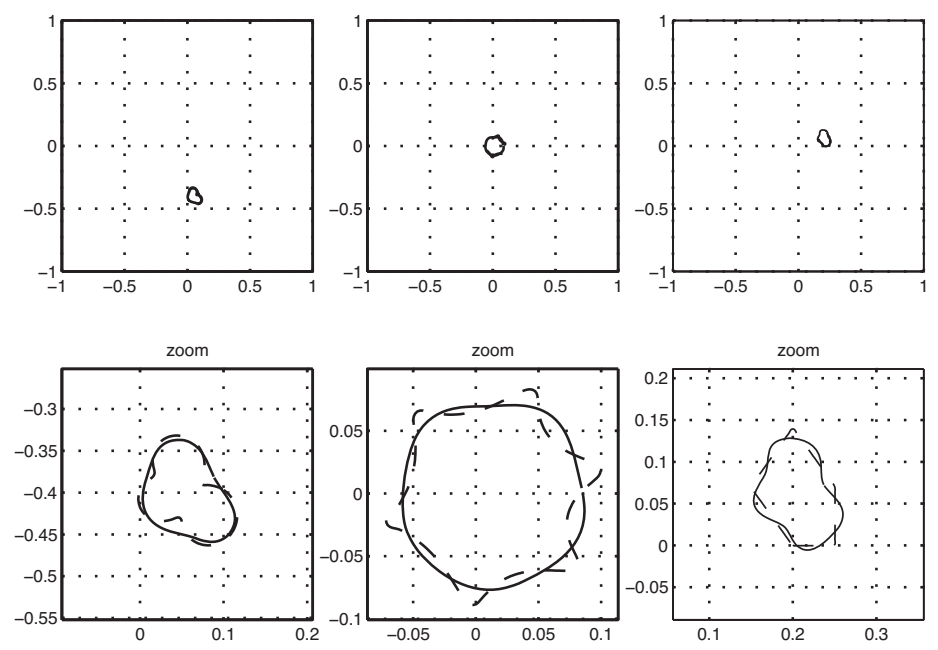

\begin{tabular}{|c|c|c|c|}
\hline$(\mu, \epsilon)$ & $(36,25)$ & $(49,9)$ & $(36,25)$ \\
$\left(\mu_{e}, \epsilon_{e}\right)$ & $(48.3763,25.0453)$ & $(96.6909,9.0361)$ & $(41.6099,25.0636)$ \\
$s_{\text {stop }}$ & 3 & 5 & 5 \\
\hline Example & $(\mathrm{a})$ & $(\mathrm{b})$ & $(\mathrm{c})$ \\
\hline
\end{tabular}

FiguRe 7. Computed shapes for three general shaped targets marked with dashed-dotted curves. The solid ellipse are computed by the optimization algorithm after iterating $s$ from 1 to $s_{\text {stop }}$. We take $\left(\mu_{0}, \epsilon_{0}\right)=(4,1)$ in all the plots. Bottom figures are 6.67 times zoom-ups of the corresponding results. 

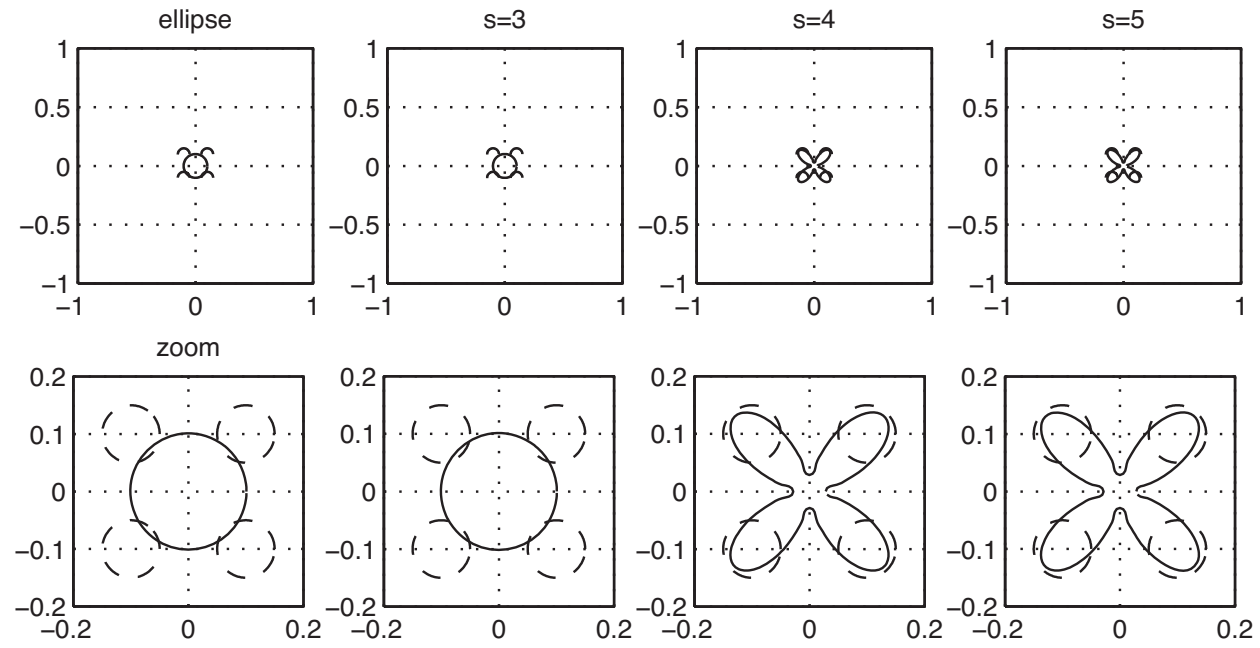

FIGURE 8. Reconstructions of multiple inclusions.
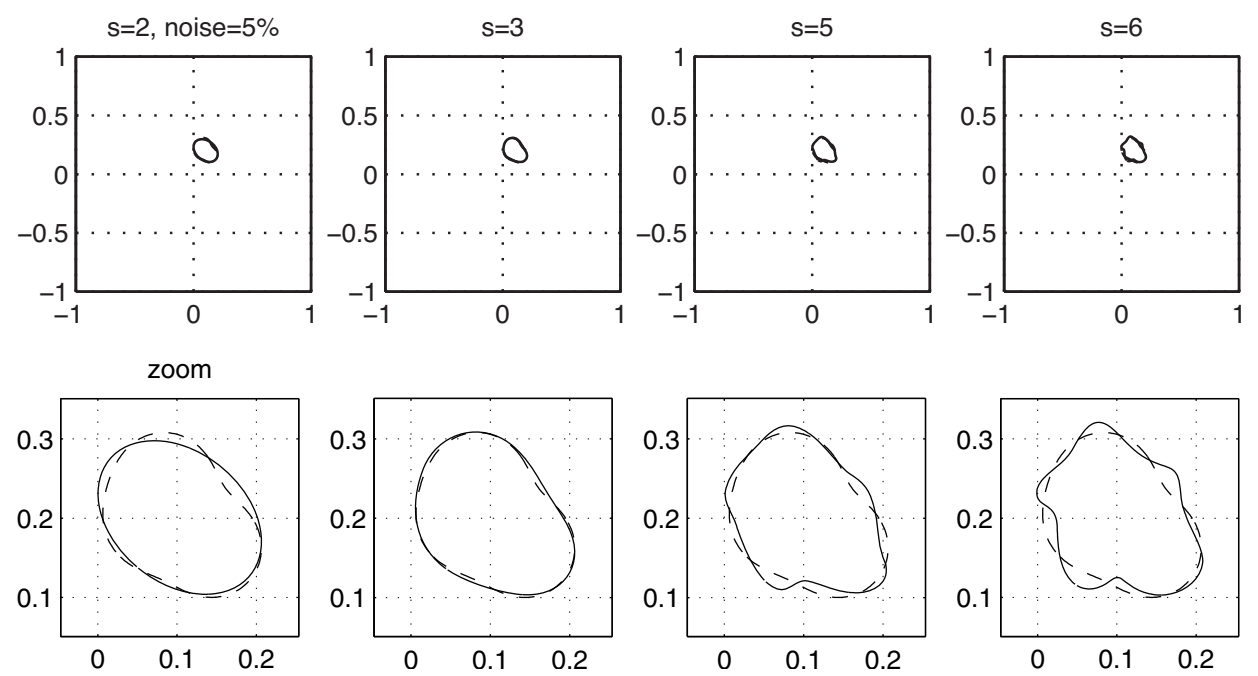

FiguRE 9. Reconstructions with 5\% additive noise. 

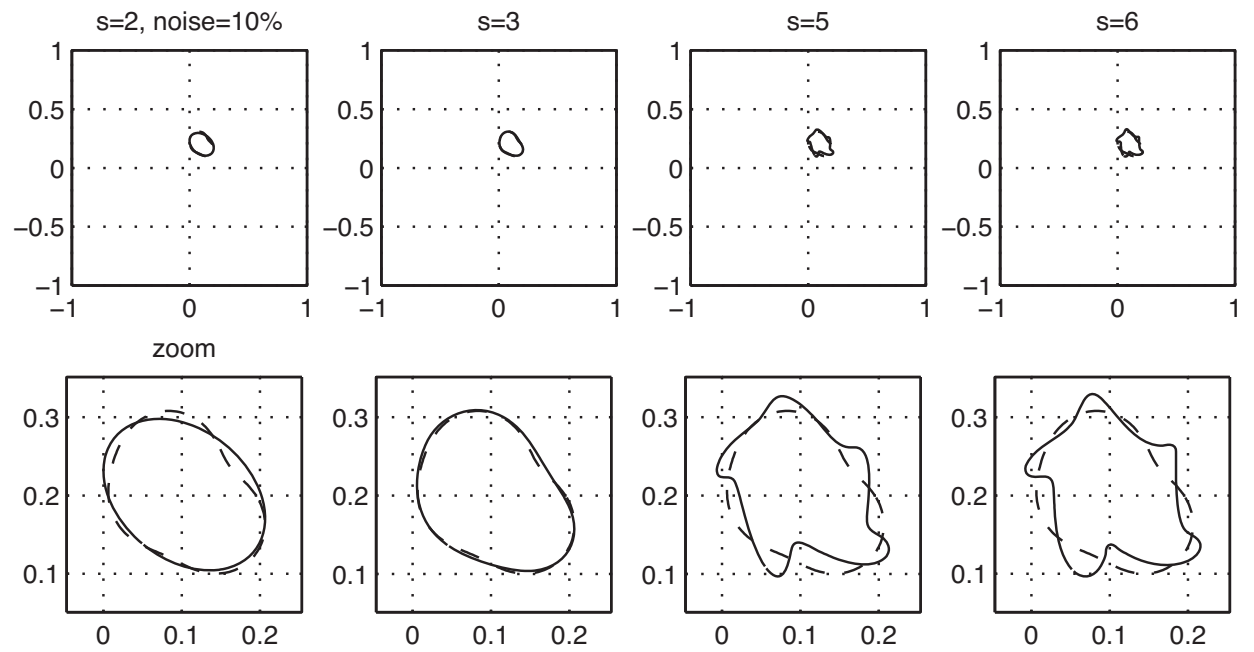

FIGURE 10. Reconstructions with $10 \%$ additive noise.

\section{Conclusion}

In this paper we have presented for the first time efficient algorithms to reconstruct from MSR measurements both electromagnetic parameters and fine shape details of a target of size smaller than the wavelength. Although, the characteristic size of the target is assumed to be smaller than the operating wavelength, a superresolved reconstruction of the shape of the target can be achieved provided a high signal-to-noise ration in the measured data. It is expected that our approach in this paper would lead to reconstruction of elastic targets as well. Another interesting extension would be the use of MSR measurements at multiple frequencies. This would be the subject of a forthcoming work. We will also attempt to precisely quantify the resolution and the stability of the proposed algorithms in terms of the signal-to-noise ratio MSR measurements.

\section{ACKNOWLEDGEMENTS}

The authors are very grateful to the reviewers for their comments and suggestions to improve the presentation of the paper. The work of HA was supported in part by the Agence Nationale de la Recherche; HK by the National Research Foundation of Korea through grants, NRF-2009-0085987 and NRF-2010-0017532; EK by the Korea Research Foundation, KRF-2008-359-C00004; and JYL by the Priority Research Centers Program, NRF-2009-0093827.

\section{REFERENCES}

[1] H. Ammari, J. Garnier, H. Kang, M. Lim, and K. Solna, Multistatic imaging of extended targets, SIAM J. Imag. Sci., to appear.

[2] H. Ammari, J. Garnier, H. Kang, W.-K. Park, and K. Solna, Imaging schemes for perfectly conducting cracks, SIAM J. Appl. Math., 71 (2011), 68-91. 
[3] H. Ammari, E. Iakovleva, and D. Lesselier, Two numerical methods for recovering small electromagnetic inclusions from scattering amplitude at a fixed frequency, SIAM J. Sci. Comput., 27, 130-158 (2005). MR2201178(2006h:35270)

[4] H. Ammari, E. Iakovleva, and D. Lesselier, A MUSIC algorithm for locating small inclusions buried in a half-space from the scattering amplitude at a fixed frequency, SIAM Multiscale Model. Simul., 3, 597-628 (2005). MR2136165 (2006e:35336)

[5] H. Ammari, E. Iakovleva, D. Lesselier, and G. Perrusson, A MUSIC-type electromagnetic imaging of a collection of small three-dimensional inclusions, SIAM J. Sci. Comput., 29 (2007), 674-709. MR2306264 (2008c:78028)

[6] H. Ammari and H. Kang, Reconstruction of Small Inhomogeneities from Boundary Measurements, Lecture Notes in Mathematics, Vol. 1846, Springer-Verlag, Berlin, 2004. MR2168949 (2006k:35295)

[7] H. Ammari and H. Kang, Boundary layer techniques for solving the Helmholtz equation in the presence of small inhomogeneities, J. Math. Anal. Appl., 296 (2004), 190-208. MR2070502 (2005c:35054)

[8] H. Ammari and H. Kang, Polarization and Moment Tensors: with Applications to Inverse Problems and Effective Medium Theory, Applied Mathematical Sciences, Vol. 162, SpringerVerlag, New York, 2007. MR2327884 (2009f:35339)

[9] H. Ammari, H. Kang, M. Lim, and H. Zribi, The generalized polarization tensors for resolved imaging. Part I: Shape reconstruction of a conductivity inclusion, Math. Comp., 81 (2012), 367-386.

[10] H. Ammari, H. Kang, E. Kim, K. Louti, and M. Vogelius, A MUSIC-type algorithm for detecting internal corrosion from electrostatic boundary measurements, Numerische Mathematik, 108 (2008), 501-528. MR2369202 (2009j:35375)

[11] H. Ammari, H. Kang, E. Kim, M. Lim, and K. Louati, Ultrasound imaging of internal corrosion, SIAM J. Numer. Anal., to appear.

[12] H. Ammari, H. Kang, H. Lee, and W.K. Park, Asymptotic imaging of perfectly conducting cracks, SIAM J. Sci. Comput., 32 (2010), 894-922. MR2609345

[13] H. Ammari and A. Khelifi, Electromagnetic scattering by small dielectric inhomogeneities, J. Math. Pures Appl., 82 (2003), 749-842. MR2005296 (2005a:35262)

[14] G. Bao and P. Li, Numerical solution of an inverse medium scattering problem for Maxwell's equations at fixed frequency, J. Comput. Phys., 228 (2009), 4638-4648. MR 2531912 (2010g:78022)

[15] L. Borcea, G. Papanicolaou, and F.G. Vasquez, Edge illumination and imaging of extended reflectors, SIAM J. Imaging Sciences, 1 (2008), 75-114. MR2475826 (2010a:15028)

[16] M. Brühl, M. Hanke, and M.S. Vogelius, A direct impedance tomography algorithm for locating small inhomogeneities, Numer. Math., 93 (2003), 635-654. MR.1961882 (2004b:65169)

[17] O. Dorn, H. Bertete-Aguirre, and G.C. Papanicolaou, Adjoint fields and sensitivities for 3D electromagnetic imaging in isotropic and anisotropic media, Inverse problems and imaging, 35-65, Lecture Notes in Math., Vol. 1943, Springer, Berlin, 2008. MR2528748 (2010h:86022)

[18] R. Griesmaier and M. Hanke, An asymptotic factorization method for inverse electromagnetic scattering in layered media II: A numerical study, Contemp. Math., Vol. 494, 61-79, Amer. Math. Soc., Providence, 2009. MR 2581766 (2010m:78018)

[19] S. Hou, K. Solna, and H. Zhao, A direct imaging algorithm for extended targets, Inverse Problems, 22 (2006), 1151-1178. MR2249458(2007b:78009)

[20] S. Hou, K. Solna, and H. Zhao, Imaging of location and geometry for extended targets using the response matrix, J. Comput. Phys., 199 (2004), 317-338. MR2081006 (2005k:78016)

[21] M.S. Vogelius and D. Volkov, Asymptotic formulas for perturbations in the electromagnetic fields due to the presence of inhomogeneities of small diameter, Math. Model. Numer. Anal., 34 (2000), 723-748. MR1784483 (2001f:78024)

[22] D. Volkov, Numerical methods for locating small dielectric inhomogeneities, Wave Motion, 38 (2003), 189-206. MR1994816 (2004f:78046)

[23] H. Zhao, Analysis of the response matrix for an extended target, SIAM J. Appl. Math., 64 (2004), 725-745. MR2068119 (2005a:74050) 
Department of Mathematics and Applications, Ecole Normale Supérieure, 45 Rue D'Ulm, 75005 PARis, France

E-mail address: habib.ammari@ens.fr

Department of Mathematics, Inha University, Incheon 402-751, Korea

E-mail address: hbkang@inha.ac.kr

Institute of Mathematical Sciences and Department of Mathematics, Ewha Womans University, Seoul 120-750, Korea

E-mail address: kej@ewha.ac.kr

Institute of Mathematical Sciences and Department of Mathematics, Ewha Womans University, SeOul 120-750, Korea

E-mail address: jyllee@ewha.ac.kr 